\title{
Extra-adrenal glucocorticoid synthesis in the intestinal epithelium: more than a drop in the ocean?
}

\author{
Mario Noti • Daniel Sidler • Thomas Brunner
}

Received: 15 April 2009 / Accepted: 14 May 2009/Published online: 3 June 2009

(C) Springer-Verlag 2009

\begin{abstract}
Glucocorticoids (GC) are lipophilic hormones commonly used as therapeutics in acute and chronic inflammatory disorders such as inflammatory bowel disease due to their attributed anti-inflammatory and immunosuppressive actions. Although the adrenal glands are the major source of endogenous GC, there is increasing evidence for the production of extra-adrenal GC in the brain, thymus, skin, vasculature, and the intestine. However, the physiological relevance of extra-adrenal-produced GC remains still ambiguous. Therefore, this review attracts attention to discuss possible biological benefits of extra-adrenal-synthesized GC, especially focusing on the impact of locally synthesized $\mathrm{GC}$ in the regulation of intestinal immune responses.
\end{abstract}

Keywords Intestinal glucocorticoid synthesis · Inflammatory bowel disease (IBD) - Intestinal immune homeostasis - Tumor necrosis factor $\alpha(\mathrm{TNF} \alpha)$.

Liver receptor homologue-1 (LRH-1)

\section{Maintenance of intestinal immune homeostasis - a delicate balance}

The intestinal tract exhibits the largest mucosal surface of the human body covering an area of almost $200 \mathrm{~m}^{2}$ [1]. This enormous surface is not only in constant contact with harmless commensal bacteria and food antigens but also with potentially harmful pathogens, such as viruses, bacteria, fungi or parasites. Critically, in the intestine, the

M. Noti $\cdot$ D. Sidler $\cdot$ T. Brunner $(\bowtie)$

Division of Immunopathology, Institute of Pathology,

University of Bern,

Murtenstrasse 31, P.O. Box 62, 3010 Bern, Switzerland

e-mail: tbrunner@pathology.unibe.ch outside world, colonized with various microorganisms, and the (generally sterile) inside world, i.e., the intestinal tissue, are separated only by a single layer of epithelial cells. This close contact with bacteria and tissue represents several potential problems [2]. On one hand, the epithelial barrier function is easily impaired enabling intestinal bacteria to invade the underlying tissue and potentially even the entire body, leading to life-threatening bacteremia. On the other hand, the close contact between non-self antigens and immune cells may also trigger a chronic stimulation of intestinal immune cells resulting in devastating inflammatory disorders [3]. Given the fact that the intestinal epithelial and subepithelial layer is home of approximately $70 \%$ of all lymphocytes in our body and also a large number of innate immune cells, this is not only an academic reflection but concerns a real constant threat [4]. In light of this side-byside closeness of immune cells and the intestinal flora with great immuno-stimulatory potential, the intestinal immune system has to find the appropriate balance between protective immune responses and tolerance.

A complex network of nonhematopoietic cells and highly adapted cells of the innate and adaptive immune system normally prevents the penetration of luminal antigens, commensal bacteria, and pathogens [5]. A first line of physical defense, the intestinal epithelial barrier, is formed by a single layer of intestinal epithelial cells displaying numerous physical and biochemical adaptations to separate the underlying intestinal tissue from the luminal space [6]. These include the formation of intercellular tight junctions (TJ), the release of mucus from goblet cells, and the secretion of a broad range of antimicrobial peptides. Furthermore, trans-epithelial transportation of $\operatorname{IgA}$ to the lumen further helps to protect the epithelial layer from bacterial invasion [7]. As discussed later on, intestinal epithelial cells are also a source of immunoregulatory 
factors, which participate in the control of intestinal immune responses [8]. Minimal alterations in the integrity of the intestinal epithelial barrier, evoked by various physical and pharmacological triggers, as well as primary defects in intestinal epithelial barrier proteins, may lead to the induction of severe intestinal disorders such as inflammatory bowel disease (ulcerative colitis or Crohn's disease in humans). In addition to this physical barrier, the intestinal mucosa contains an extensive and complex immune system, usually confirming hyporesponsiveness or tolerance towards commensal bacteria while maintaining the ability to fight pathogenic microorganisms $[9,10]$. Intense communication between the intestinal epithelium and cells of the innate and adaptive immune system is critical to achieve proper function of the intestinal immune system, warrant host protection, and avoid unnecessary inflammation-associated damage [11].

Dysregulation of the balance between tolerance and immunity leads to severe pathogenesis in the intestinal tract, including inflammatory bowel disease (IBD), and chronic inflammatory responses may result in the development of intestinal cancer. A hallmark of the adaptive intestinal immune system is the relatively high number of regulatory immune cells within the lamina propria. Regulatory $\mathrm{T}$ cells ( $\mathrm{T}$ reg) and the associated secretion of immunosuppressive cytokines such as IL-10 or TGF $\beta$ have been ascribed a major role in the maintenance of intestinal immune homeostasis $[12,13]$. Adoptive transfer of $\mathrm{T}$ reg not only prevents the development of experimental colitis in various animal models but also ameliorates established disease [14]. Furthermore, it has been shown that animals deficient in the production of IL-10 or TGF $\beta$ develop chronic intestinal inflammation [15]. Beside these and other well-known immunoregulatory mechanisms contributing to the maintenance of intestinal homeostasis, the synthesis of immunosuppressive glucocorticoids (GC) by intestinal epithelial cells seems to represent a novel and important mechanism in the maintenance of intestinal immune homeostasis [16]. In this review, we will discuss various aspects of GC synthesis in the intestinal epithelium and their action on local immune cells.

\section{Actions and anti-inflammatory properties of GC}

GC exert a variety of important anti-inflammatory actions. Apart their crucial role in glucose metabolism and blood pressure regulation, GC are very important regulators of immune reactions $[17,18]$. Depending on where and when GC act on target cells, the anti-inflammatory and immunoregulatory properties of GC may be mediated by different mechanisms. GC normally interact and bind to their corresponding glucocorticoid receptor (GR) in the cyto- plasm. The inactive GR is coupled to a protein complex including two subunits of the heat shock protein 90 (hsp90) acting as chaperones to prevent nuclear translocalization of unoccupied GR. Upon binding of GC, the GR dissociates from hsp90 leading to nuclear translocation of the GC/GR complex. The agonist-induced translocation of the GR to the nucleus is illustrated in Fig. 1. In the nucleus, this complex binds to specific DNA sequences in the promoter regions of target genes, so-called GC response elements [19], or to other proteins (mostly transcription factors), and thereby either increases (transactivation) or decreases (transrepression) the expression of responsive genes. GC/ GR complexes increase gene transcription through chromatin remodeling and recruitment of RNA polymerase II by interacting with co-activator proteins, such as steroid receptor co-activator-1 (SRC-1), leading to an enhancement of histone acetyl transferase activity. Local changes in DNA or chromatin configurations through GC/GR interactions, that might be previously masked, lead to an increase in binding of transcription factors and additionally to a more stable transcription initiation complex [20].

Various immunoregulatory functions of GC seem to act via distinct molecular targets. One of the most important activities of GC is their inhibitory effect on the production of pro-inflammatory cytokines, as for example IL-1 $\beta$, IL-6, IL-12, TNF $\alpha$, INF $\gamma$, and others [21, 22]. This inhibition is most probably mediated through direct interaction with the transcription factors $\mathrm{AP}-1$ or $\mathrm{NF} \kappa \mathrm{B}$ [23-25]. $\mathrm{NF} \kappa \mathrm{B}$ is activated through a number of cytokines, such as TNF $\alpha$ or IL-1 $\beta$, UV irradiation, stress, and bacterial and viral infections. The transcriptional activity of $\mathrm{NF} \kappa \mathrm{B}$ can be modulated by GC bound to the GR by physical interaction with the active trans-activating p65 RelA subunit of NFkB, leading to the hindrance of NFKB binding to the appropriate DNA recognition motifs in promoter regions of different target genes [26]. Furthermore, GC have been demonstrated to increase the rate of $\mathrm{I} \kappa \mathrm{B}$ (inhibitor of $\mathrm{NF} \kappa \mathrm{B}$ ) protein synthesis, which in turn traps the activation of NFKB. In addition, direct protein-protein interactions between GR

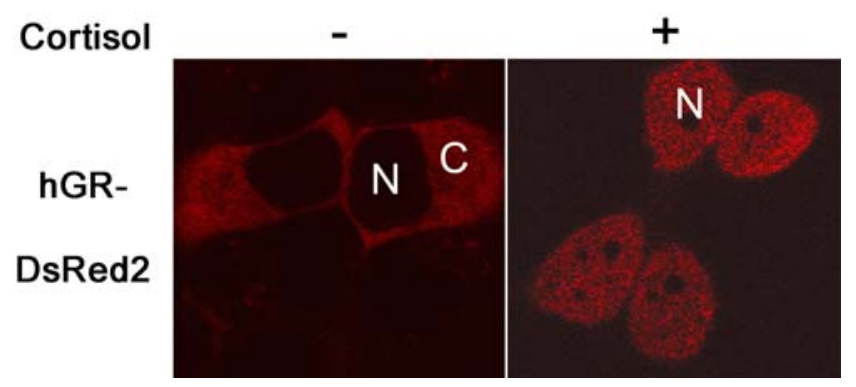

Fig. 1 Ligand-induced translocation of the glucocorticoid receptor. HEK cells were transfected with a fluorescence-labeled glucocorticoid receptor (hGR-DsRed2), and nuclear translocation was induced by addition of cortisol. $N$ nucleus, $C$ cytoplasm 
and AP-1, STAT3, STAT5, and STAT6 have been reported indicating a modulation of binding or activation of these transcription factors resulting in a modified expression of inflammatory genes [27]. Finally, it should not be overseen that GC are potent inducers of apoptosis in a variety of immune cells [28, 29]. GC-mediated apoptosis may be either induced by inhibiting the expression of NFkBinduced anti-apoptotic molecules or the direct induction of the pro-apoptotic Bcl-2 homolog Bim [30-32]. Notably, many immune cells are strongly resistant to GC-induced apoptosis in the absence of Bim [31]. Due to these immunoregulatory and anti-inflammatory properties, GC are widely used in clinical applications ranging from preventing rejection of transplants, treatment of allergic asthma and rheumatoid arthritis, to highly effective use in IBD [33-36].

\section{The adrenals: a small organ with an enormous steroid-producing capacity}

GC are lipid hormones synthesized from the precursor cholesterol through sequential conversions by members of the cytochrome P450 (CYP) superfamily and $3 \beta$ hydroxysteroid dehydrogenase $(3 \beta \mathrm{HSD})$ to cortisol in humans and corticosterone in rodents [37].

GC synthesis and release from adrenal glands is predominantly regulated by the hypothalamus-pituitaryadrenal axis [38]. The release of corticotropin-releasing hormone from the paraventricular nucleus located in the hypothalamus directly stimulates the secretion of adrenocorticotropin-releasing hormone (ACTH) in the anterior pituitary gland. ACTH reaches the adrenal cortex via the blood stream and binds to G-protein-coupled ACTH receptors on the surface of cells in the zona reticularis. ACTH induces the activation of adenylate cyclase leading to a subsequent increase in intracellular cAMP levels having pleiotropic functions such as the activation of protein kinase A [39]. cAMP response elements (CRE) in turn are found in the promoter region of a variety of steroidogenic genes, mediating GC synthesis induction in response to these pathways [40]. Adrenal GC synthesis is able to respond very rapidly to a variety of triggers, often within minutes, and thus provides the body with appropriate levels of GC. Adrenal-derived GC are crucially involved in glucose metabolism, insulin release for blood sugar maintenance, regulation of blood pressure, as well as the maintenance of immune homeostasis [41]. Therefore, adrenal insufficiency, characterized by lack of endogenous GC synthesis as observed in Addison disease, or adrenalectomy results in hypoglycemia, hypotension, and increased susceptibility to inflammatory and autoimmune disorders [42]. On the other hand, chronic treatment with therapeutic doses of GC results in immune suppression and opportunistic infections with microorganisms. The protective effect of endogenous GC against the lethal effect of high-dose endotoxins, IL- $1 \beta$, and TNF $\alpha$ has been known for a long time. Thus, not surprisingly, adrenalectomy or inhibition of systemic GC synthesis by specific inhibitors results in rapid death by shock after induction of strong immune responses [43-45].

\section{GC-not only produced in the adrenals}

The lack of serum GC in adrenalectomized animals strongly supports the role of adrenal glands as the major source of GC, at least for those GC that can be measured in the blood stream. There is however an increasing number of studies and experimental evidence that points towards alternative sources of GC. Extra-adrenal GC synthesis was initially described in the thymic epithelium, mostly by work of Jonathan Ashwell and colleagues, and was later extended to other organs [46]. Interestingly, although thymocytes rapidly die by apoptosis in response to exogenous and endogenous GC, in vivo and ex vivo experiments of Ashwell and colleagues suggested that local thymic GC synthesis is important for positive selection by lowering the activation signal induced by the $\mathrm{T}$ cell receptor [46, 47]. These findings have been questioned by others though, and the definite role of thymic GC synthesis remains to be further investigated. However, clearly the thymic epithelium expresses all the steroidogenic enzymes required to synthesize GC and thus represents an extra-adrenal source of GC [48, 49].

Subsequently, other tissues such as brain, skin, and vascular endothelium have been described to express steroidogenic enzymes and were claimed to be potential extra-adrenal sources of GC [50]. So far, it has been difficult to investigate the role of extra-adrenal GC synthesis in these organs in vivo and to determine its relevance for health and disease. A major problem in the investigation of extraadrenal GC synthesis is the fact that the adrenals, although a tiny organ, are the most potent source for GC, which are distributed to all peripheral tissues via the circulation. Therefore, it is often difficult to distinguish between serum contamination and local steroidogenesis in a given tissue. Furthermore, it is very difficult to pharmacologically block local GC synthesis while maintaining proper adrenal function. In the future, the tissue-specific deletion of steroidogenic enzymes may help to better define the role of extra-adrenal GC synthesis in non-adrenal tissues and determine its role for health and disease. Nonetheless, in certain tissues, such as the intestine, investigations of extraadrenal GC synthesis have been possible in various experimental models. These experiments have helped to 
reveal the important role of local GC synthesis in fine-tuning local immune responses [16].

\section{Intestinal epithelial cells as source of extra-adrenal GC}

Accumulating evidence for the specific role and regulation of local GC synthesis is available for the intestinal mucosa. Already in 1995 it was reported by Keith Parker and colleagues that the cholesterol side-chain cleavage enzyme P450scc (CYP11A1), converting cholesterol to pregnenolone, is expressed in the primitive gut of the mouse embryo, suggesting that the intestinal mucosa may represent a steroidogenic organ [51]. Later on, further evidence was provided by a study from our lab for the synthesis and action of intestinal GC. Intra-epithelial lymphocytes (IEL) are $\mathrm{T}$ cells with a predominantly unconventional phenotype that reside within the intestinal epithelial layer in direct physical contact with the epithelial cells. Although most of the IEL are CD8+, they show no cytotoxic potential but rather exhibit a regulatory phenotype. Analysis of IEL effector function is severely hampered by their rapid death when cultured ex vivo. While studying the mechanisms of IEL apoptosis, it was noticed that IEL are very sensitive to GC-induced apoptosis and that in vivo treatment of mice with the GR antagonist RU-486 significantly reduced ex vivo apoptosis of IEL. In contrast, adrenalectomy only weakly reduced IEL apoptosis, indicating that an alternative source of GC, likely within the intestinal mucosa, may be responsible for triggering IEL cell death [52].

As mentioned above and shown in Fig. 2, GC, such as corticosterone and cortisol, are produced from cholesterol via specific enzymatic pathways. Interestingly, in the murine small or large bowel, only some of the steroidogenic enzymes are constitutively expressed. Critically, some genes encoding for these enzymes, in particular CYP11A1 (side chain-cleaving enzyme, P450scc) and CYP11B1 (11ßhydroxylase), are strongly induced upon activation of immune cells. Notably, injection of an agonistic anti-CD $3 \varepsilon$ antibody, leading to a rapid activation of $\mathrm{T}$ cells, results in a strong upregulation of several steroidogenic enzymes in the intestinal tissue within few hours, suggesting a counterregulatory reaction of the local tissue in response to strong immune cell activation and initiation of inflammatory processes. Upon immune cell activation, not only the expression of steroidogenic enzymes is induced, but steroidogenesis is actually initiated. Tissue samples from small and large intestine can be isolated and cultured for a limited time ex vivo. Interestingly, corticosterone produced in these tissue samples is easily detectable by radioimmunoassay or gas chromatography/mass spectrometry in the culture supernatant. Importantly, GC measured are bona fide produced within these intestinal tissue samples, as their synthesis is

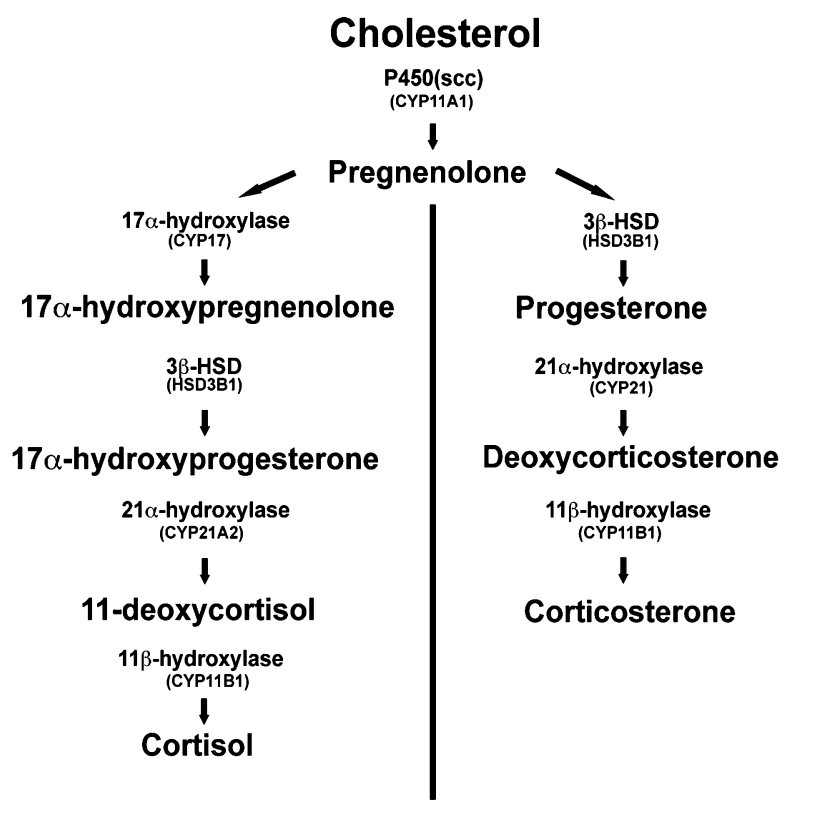

human steroidogenesis murine steroidogenesis

Fig. 2 Simplified scheme of adrenal glucocorticoid synthesis in humans and rodents

blockable by addition of metyrapone, a specific inhibitor of $11 \beta$-hydroxylase and thus GC synthesis [16].

What is the relevant cellular source of intestinal GC? The intestinal mucosa is a complex tissue comprising a variety of different cell types. As in the adrenal glands, GC are produced in epithelial cells: epithelial cells in the intestinal mucosa are also a likely source of GC. Indeed, in situ hybridization experiments as well as differential isolation of cells from the intestinal mucosa revealed that the expression of steroidogenic enzymes is defined to the intestinal epithelial layer and more specifically to the intestinal crypts $[16,53,54]$. As discussed later on, this localized expression of steroidogenic enzymes is also relevant for the adapted regulation of intestinal GC synthesis, in comparison to that in the adrenal glands.

\section{Differences in the regulation of adrenocortical versus intestinal GC synthesis}

Whereas the regulation of adrenal GC synthesis has been the topic of extensive studies and many of the signaling pathways involved have been identified so far, little is known regarding the regulation of extra-adrenal GC synthesis. Mueller et al. recently pointed out that the molecular basis of GC synthesis in intestinal epithelial cells is substantially distinct from that in adrenal cells, most likely indicating an adaptation to the different local settings and requirements [55]. First evidence for a local adapted intestinal GC synthesis is the differential expression of the two orphan nuclear receptors SF-1 (steroidogenic factor-1, NR5a1) and its close homologue 
LRH-1 (liver receptor homologue-1, NR5a2). SF-1 is a critical transcription factor in the regulation of adrenal GC synthesis [56]. Absence of SF-1 results in lack of adrenal steroidogenesis and thereby in the absence of serum GC [57]. Not surprisingly, SF-1 is abundantly expressed in the adrenal gland. In sharp contrast, the intestine does not express SF-1, but it seems to be functionally replaced by its close homologue LRH-1 [55, 58]. Already previously, it has been shown that CYP11A1 expression in the murine embryonic gut is not dependent on SF-1, suggesting that other transcription factors regulate the expression of steroidogenic enzymes in this organ [51]. LRH-1 and SF-1 have a high sequence homology, overlapping activities, and bind to the same consensus sequences in the promoter regions of steroidogenic enzymes [59]. LRH-1, almost not detectable in the adrenal glands, is abundantly expressed in the intestinal crypts, where it regulates local GC synthesis. In agreement with this role of LRH-1, it was found that overexpression of LRH-1 in intestinal epithelial cells is sufficient to induce the expression of steroidogenic enzymes and trigger GC synthesis in vitro. More critically, LRH-1 haploinsufficiency (complete deficiency is embryonically lethal [60]) severely impairs intestinal GC synthesis, e.g., as induced by injection of anti-CD3 $\varepsilon$ antibodies, whereas adrenal GC synthesis and systemic GC levels remain unaffected [61].

This differential expression and use of critical regulators of steroidogenesis in the adrenals versus the intestinal epithelium, i.e., SF-1 versus LRH-1, strongly argues for a substantially different regulation of the adrenal versus the intestinal GC synthesis. It further points out that this differential regulation is likely the result of different needs for the systemic and the intestinal GC synthesis. Clearly, adrenal GC synthesis must be very rapid and very strong to provide the body with sufficient levels of GC within a minimal amount of time. In contrast, velocity of intestinal GC synthesis may not be so critical but rather the appropriateness of its induction to allow or prevent local immune responses. Furthermore, constitutive low levels of intestinal GC may also be required to maintain optimal intestinal barrier functions. It is thus interesting to note that $\mathrm{GC}$ regulate the expression of tight junction proteins $[62,63]$.

A major difference in the regulation of intestinal versus adrenal GC synthesis is the respective role of the cAMP signaling pathway. It is well established that adrenal steroidogenesis is critically regulated by the peptide hormone ACTH [64]. The activation of the ACTH receptor leads to the activation of the adenylate cyclase and the formation of cAMP [65]. This in turn is critical for the activation of protein kinase $A$ and subsequently the induction of a variety of steroidogenic enzymes, in particular of the cytochrome P450 family, via so-called CRE in their promoters [66]. The ACTH signaling pathway can be easily mimicked by treating cells with cell-permeable cAMP (e.g.,
8-bromo-cAMP) or forskolin, which leads to an increase in intracellular cAMP levels. Surprisingly, while these triggers induce the expression of steroidogenic enzymes and $\mathrm{GC}$ synthesis in adrenal cell lines, they have completely the opposite action in intestinal epithelial cells. Instead of inducing steroidogenesis, cAMP treatment even leads to an inhibition of basal and LRH-1-driven GC synthesis. A reciprocal finding is observed when treating the cells with phorbol ester. Phorbol ester, such as phorbol myristate acetate (PMA), are well-known activators of protein kinases, e.g., members of the protein kinase $\mathrm{C}$ family. Interestingly, in adrenal cells, PMA has little effect on steroidogenesis or even an inhibitory action. In marked contrast, PMA treatment enhances both basal as well as LRH-1-driven expression of steroidogenic enzymes and GC synthesis in intestinal epithelial cells [55]. Likely, this is due to specific activation of LRH-1. Although crystal structure analysis of LRH-1 revealed putative ligands (e.g., phospholipids) whose binding might regulate LRH-1 transcriptional activity, at least murine LRH-1 can be activated ligand-independently [39, 67]. In contrast, the MAP kinase-mediated phosphorylation of the hinge region of LRH-1 appears to be important for the regulation of LRH-1 activity [68]. As PMA is a potent activator of the MAP kinase pathway, it is likely that PMA affects LRH-1 activity by inducing its phosphorylation [69]. While the actual molecular mechanism of cAMP-mediated inhibition and PMA-induced activation of intestinal GC synthesis are currently not fully understood, these findings further support the different requirements of and regulation of intestinal versus adrenal steroidogenesis.

\section{LRH-1, cell cycle, and GC synthesis}

LRH-1 has not only been implicated in the regulation of steroidogenesis but also in other cellular processes. Of note is a recent finding by Botrugno and colleagues who reported that LRH-1 may be critical for intestinal crypt cell proliferation by inducing cyclin D1 and E1 expression [70]. Not surprisingly, it was further found that LRH-1 may also promote the development of intestinal epithelial tumors [71]. Thus, LRH1 appears to regulate intestinal epithelial layer integrity via two distinct processes. Upon epithelial damage, LRH-1induced proliferation of crypt cells may help to close the gaps and to recover intestinal epithelial barrier functions. In agreement with its cell cycle-regulating role, we observed that LRH-1 is predominantly expressed in the proliferating cells of the crypts and is lost in the differentiated cells of the villus [53]. On the other hand, LRH-1 likely contributes to epithelial layer repair by the regulation of intestinal GC synthesis, thereby inhibiting inflammatory process and inducing TJ proteins [62] (Fig. 3). 


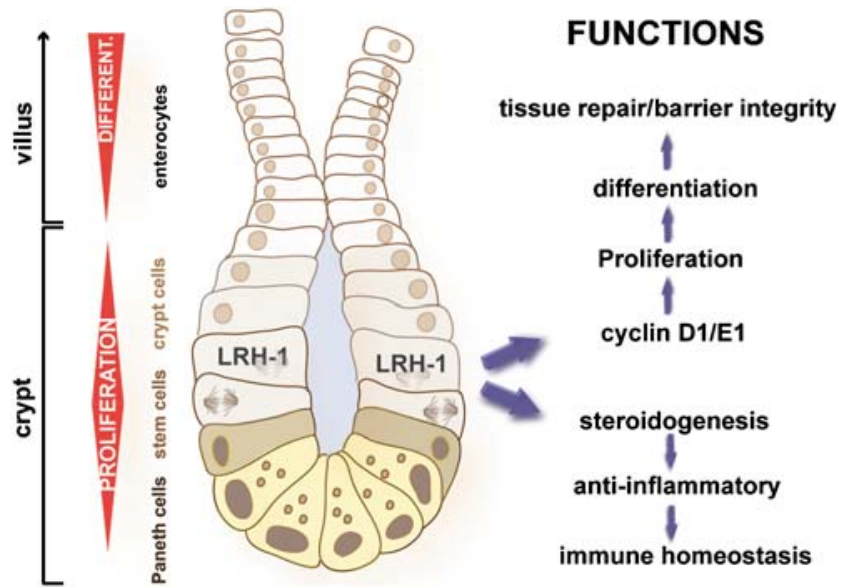

Fig. 3 The role of LRH-1 in intestinal epithelial cells. A simplified scheme of the intestinal crypt region illustrates the dual role of LRH-1 in the intestine by promoting cell proliferation to maintain epithelial barrier integrity and by inducing intestinal steroidogenesis

There is yet another interesting connection between LRH-1 and cell cycle. Clearly, LRH-1 should exclusively be active in the crypt region in order to prevent uncontrolled cyclin expression and cell cycle progression, and thereby promote tumorigenesis. A similar pattern is seen when LRH-1-induced steroidogenesis is assessed. Inhibition of cell cycle progression by either pharmacological or biological cell cycle inhibitors not only blocks cell cycle progression of crypt cell-like epithelial cells but also strongly inhibits LRH-1 transcriptional activity, the expression of steroidogenic enzymes, and the synthesis of GC. Similarly, epithelial cell differentiation seems to limit the activity of LRH-1 and thereby the transcription of target genes such as cyclin D1, E1, and steroidogenic enzymes. For example, the differentiation of the colonic crypt cell line YAMC leads to cell cycle arrest, an upregulation of the cell cycle inhibitor $\mathrm{p} 21$ and a downregulation of cyclin D1 and CYP11B1 [53]. This in vitro finding probably recapitulates what is likely to happen when undifferentiated crypt cells differentiate to more mature absorptive epithelial cells. Indeed, LRH-1, cyclin D1, and steroidogenic enzyme expression is largely restricted to the proliferating cells of the crypts and gets gradually lost towards the villus tip [16, 53]. This effect may largely restrict the expression of steroidogenic enzymes and intestinal GC synthesis to the self-renewing pool of epithelial stem cells and thereby further help to maintain epithelial layer integrity.

\section{Intestinal GC synthesis and the regulation of local immune responses}

Due to their potent anti-inflammatory actions, GC have important therapeutic applications in the treatment of inflammatory disorders, including those of the intestinal mucosa. Thus, GC are first-line therapeutic agents for the treatment of ulcerative colitis (UC) and Crohn's Disease (CD) [72]. Independently of the route of application, GC rapidly improve the outcome of moderate to severe acute $\mathrm{UC}$ and $\mathrm{CD}$, most probably by dampening overwhelming immune responses in the inflamed intestine. Also in the normal mucosa, the intestinal immune system is confronted with the difficult task to protect the intestinal mucosa while maintaining immune homeostasis and to avoid overshooting immune responses. These processes are tightly regulated by a variety of mechanisms. Intestinal epithelial cells appear to critically contribute to the maintenance of intestinal immune homeostasis via the production of extraadrenal GC [8].

Role of local GC synthesis in the regulation of intestinal antiviral immune responses

Viral infections often elicit strong and protective immune responses, which may however also result in tissue destruction and immunopathology. This is particularly evident in the liver where the antiviral immune response rather than the viral infection per se leads to hepatitisassociated tissue damage [73]. The local production of GC may help to dampen such strong immune responses and to protect the tissue from uncontrolled damage. The hypothesis that intestinal GC synthesis may regulate local antiviral immune responses was recently tested by Cima et al. [16]. Infection of mice with lymphocytic chorionmeningitis virus leads to rapid spreading and replication of the virus in various organs, including the intestinal mucosa. The strong activation and expansion of cytotoxic antiviral CD8+ T cells is subsequently responsible for the elimination of virus-infected cells and the clearance of the virus. Employing this system, it was noted that in the absence of local GC synthesis, the activation of virus-specific intestinal $\mathrm{T}$ cells was significantly more pronounced. Antigen-specific $T$ cells isolated from the epithelial layer, the lamina propria as well as from Peyer's patches expressed significantly higher levels of the activation marker CD69 and produced more IFN $\gamma$ when local GC synthesis was blocked. Thus, locally produced GC seem to act on gut-homing antigenspecific $T$ cells in a paracrine manner by substantially buffering their activation potential.

Intestinal GC synthesis during inflammatory bowel disease

In general viral infections, antiviral immune responses and associated immunopathologies are only transient. Much in contrast, uncontrolled inflammatory disorders of the intestinal mucosa often result in chronic self-amplifying disorders, e.g., as observed in IBD. It is thus of major interest to understand to which extent intestinal GC synthesis 
contributes to the regulation of the pathogenesis of IBD. Coste et al. recently investigated this issue indirectly by investigating the role and impact of LRH-1 in the regulation of experimental IBD and in human IBD patients [61]. The induction of experimental colitis in wild-type mice led to a substantial upregulation of mRNA transcripts for steroidogenic enzymes as well as to a clear induction of in situ synthesized intestinal GC, suggesting intestinal GC synthesis as part of a negative feedback response. In contrast, steroidogenic enzyme expression and intestinal GC synthesis was significantly reduced in LRH-1 haplo-insufficient mice or mice with tissue-specific deletion of LRH-1, confirming the important role of LRH-1 in the regulation of intestinal GC synthesis. Importantly, absence of LRH-1 in the intestinal epithelium led to an increased susceptibility to dextran sodium sulfate (DSS)- and TNBS-induced colitis and an exacerbation of immunopathology. Colitogenic LRH-1-deficient mice were characterized by increased infiltration of mononuclear cells and a boost in colonic pro-inflammatory cytokine mRNA expression compared to wild-type controls. Interestingly, these experimental data could be confirmed by expression data in human patients with UC and CD, in which reduced levels of LRH-1 and steroidogenic enzymes, but increased expression of proinflammatory cytokines, were observed in colonic biopsies of inflamed tissue compared to healthy tissue [61].

In line with the results of Coste et al., we recently described the induction of steroidogenic enzymes and associated GC synthesis in different animal models of colitis, i.e., DSS-, TNBS-, and oxazolone-induced colitis. Interestingly, the induction of colitis-associated GC synthesis seems not to be dependent on the induction of inflammation per se but on the type of inflammation. Whereas all three models of colitis led to profound colonic inflammation with massive leukocyte infiltration and epithelial layer damage, the induction of intestinal GC synthesis was quite distinct in the different model systems. Whether or not intestinal steroidogenesis was induced seemed to depend on the cytokine profile produced and more specifically on the presence of specific proinflammatory cytokines. Thus, DSS and TNBS, which induce a Th1 cytokine profile, promoted intestinal GC synthesis, whereas oxazolone, inducing a Th2 cytokine profile, failed to do so (Noti M, Brunner T, unpublished data).

$\mathrm{TNF} \alpha$ as critical regulator of intestinal GC synthesis

$\mathrm{TNF} \alpha$ is a well-characterized pro-inflammatory cytokine with a critical role in the pathogenesis of inflammatory bowel disease (IBD) [74]. In particular, TNF $\alpha$ appears to be important for the initiation of the intestinal inflammation [75]. Consequently, absence or neutralization of TNF $\alpha$ ameliorates disease progression in different (but not all) experimental colitis models and human patients [76, 77]. Treatment of IBD patients with the neutralizing anti-TNF $\alpha$ antibody Infliximab has astonishing beneficial effects and is surprisingly long lasting. This has led to the hypothesis that Infliximab may not only neutralize soluble TNF $\alpha$ but likely also target transmembrane TNF $\alpha$-expressing immune cells by inducing cell death [78]. Clearly, these findings illustrate the potent pro-inflammatory properties of TNF $\alpha$ in IBD.

Apart the well-known pro-inflammatory actions of $\mathrm{TNF} \alpha$, there is increasing evidence for various antiinflammatory effects of this cytokine [76, 79]. While part of this activity was attributed to direct apoptosis induction or sensitization to apoptosis induction by other triggers, other mechanisms are also likely involved [80, 81]. We and others recently showed that lack of TNF $\alpha$ exacerbates disease progression in DSS-induced colitis ([76] and unpublished data). TNF $\alpha$ seems to have a protective effect in DSS-induced colitis as absence of TNF $\alpha$ leads to increased weight loss and significantly higher colonic myeloperoxidase levels, indicative of neutrophilic infiltration, compared to DSS-treated wild-type animals. While in wild-type animals the onset of the disease was paralleled by a time-dependent increase in colonic GC synthesis, steroidogenic enzyme expression and associated intestinal GC synthesis were drastically reduced in $\mathrm{TNF} \alpha$-deficient animals despite the pronounced induction of colonic inflammation. The importance of $\mathrm{TNF} \alpha$ in the induction of intestinal GC synthesis could be confirmed by a significant reduction in steroidogenic enzyme expression and intestinal GC synthesis in TNF $\alpha$-deficient animals in response to strong in vivo $\mathrm{T}$ cell activation, i.e., injection of the agonistic anti-CD $3 \varepsilon$ antibody. Similarly, direct injection of $\mathrm{TNF} \alpha$ in healthy animals leads to a pronounced induction of intestinal GC synthesis, confirming its direct activity on intestinal steroidogenesis (Noti M, Brunner T, unpublished data; Fig. 4).

This novel view of the anti-inflammatory activity of TNF $\alpha$ also opens new perspectives. As discussed above, a Th2 cytokine-mediated experimental form of colitis induced by oxazolone failed to promote colonic GC synthesis. This is likely due to the absence of TNF $\alpha$ and an overproduction of Th2 cytokines, such as IL-4. Remarkably, therapeutic treatment of mice with oxazolone colitis with recombinant $\mathrm{TNF} \alpha$ restores steroidogenic enzyme expression, colonic GC synthesis, and significantly ameliorates the clinical parameters in treated animals. While $\mathrm{TNF} \alpha$ has been previously associated with the induction of cachexia [82] and was therefore considered an inducer of body weight loss, daily treatment of colitogenic mice with recombinant murine $\mathrm{TNF} \alpha$ significantly reduced oxazolone-induced body weight loss and led to overall 


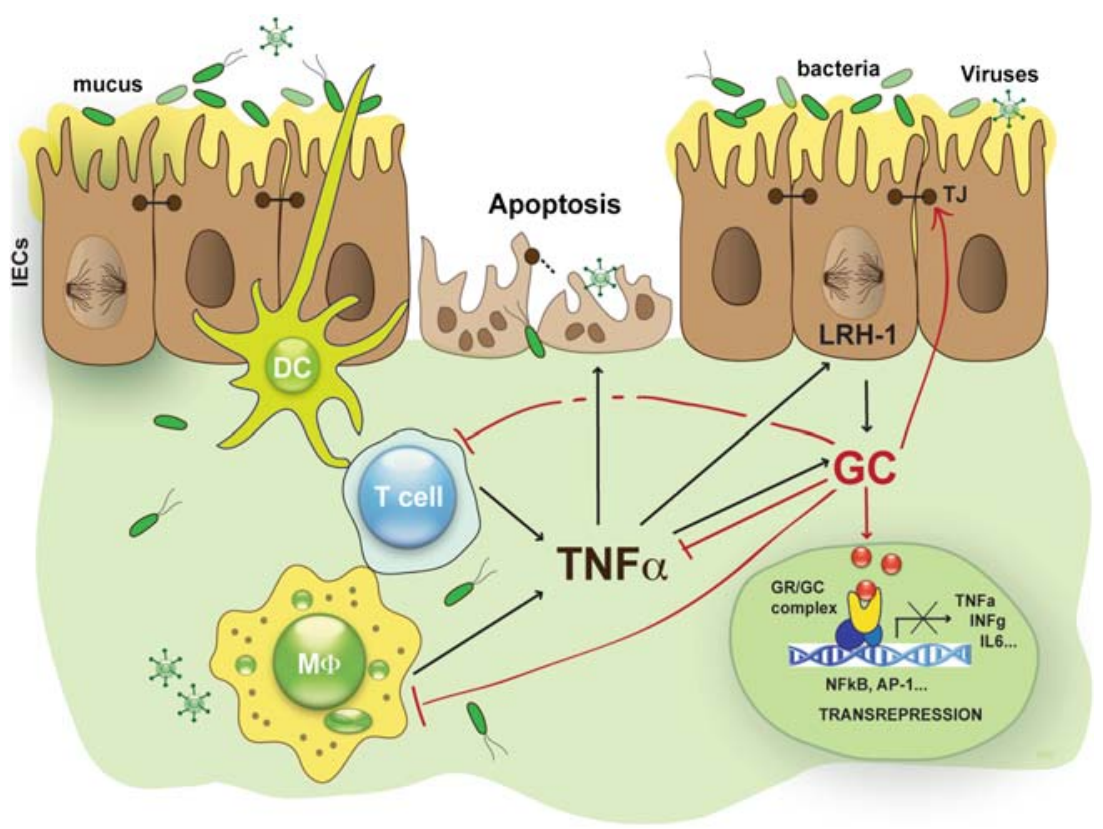

Fig. 4 Regulation of intestinal glucocorticoid synthesis by $\mathrm{TNF} \alpha$. Alterations in epithelial barrier integrity lead to the activation of intestinal immune cells releasing pro-inflammatory cytokines such as $\mathrm{TNF} \alpha$, which further amplifies the local immune response and associated immune cell-mediated tissue damage. Intestinal epithelial cells counter-regulate an overwhelming immune response by releasing immunoregulatory factors. TNF $\alpha$ directly induces steroidogenesis in

improved tissue pathology and colitis score. Thus, TNF $\alpha$ appears to have a novel, rather paradoxical, antiinflammatory activity in the intestinal mucosa via the induction of GC synthesis in intestinal epithelial cells. Importantly, so far all previously described antiinflammatory properties of TNF $\alpha$ have been attributed to act on $\mathrm{T}$ cells. These findings now provide evidence for an alternative pathway via the induction of intestinal steroidogenesis. While experiments with epithelial cell lines in vitro prove that $\mathrm{TNF} \alpha$ can directly induce the expression of steroidogenic enzymes in these cells, the exact mode of action and associated mechanisms of TNF $\alpha$-induced or $\mathrm{TNF} \alpha$-mediated intestinal GC synthesis remain to be elucidated but offer novel opportunities for therapeutic intervention.

GC and their beneficial effect on intestinal epithelial barrier integrity

While intestinal GC likely have an immunoregulatory activity on local and infiltrating immune cells and thus on the development of intestinal inflammation, intestinal GC synthesis may affect local inflammation also by other means. Therapeutic administration of GC has been reported to induce intestinal tight junction (TJ) proteins and improve epithelial barrier functions in CD patients. Normalization of the intestinal permeability correlated directly with clinical intestinal epithelial cells leading to the secretion of immunoregulatory glucocorticoids. Glucocorticoids regulate the activation of transcription factors, such as AP-1 or NFKB (transrepression), leading to reduced production of pro-inflammatory cytokines. $D C$ dendritic cells, $G C$ glucocorticoids, $G R$ glucocorticoid receptor, $M \Phi$ macrophage, $T J$ tight junction

amelioration of the disease and is a positive predictor of prolonged clinical remission [23, 40]. As colitis affects severely the intestinal epithelium, Boivin et al. investigated the role of exogenously administered GC on the integrity of the intestinal epithelial TJ by using an intestinal epithelial cell line [62]. Strikingly, they observed that, in Caco-2 cells, GC inhibited TNF $\alpha$-induced increase in myosin light chain kinase (MLCK) expression, which plays a major role in mediating increased intestinal $\mathrm{TJ}$ permeability. It has been previously shown that myosin light chain phosphorylation by MLCK is responsible for TJ disruption and increased epithelial layer permeability. Both genetic and pharmacological MLCK inhibition prevented in vivo epithelial barrier dysfunction following systemic $\mathrm{T}$ cell activation [83]. Local intestinal GC synthesis, as induced upon induction of intestinal inflammation, may therefore attenuate TNF $\alpha$-mediated TJ barrier disruption by inhibiting $\mathrm{TNF} \alpha$-induced MLCK expression. Thus, TNF $\alpha$ may again play a dual role in intestinal inflammation, on one hand a pro-inflammatory by inducing immune cell activation and disrupting the epithelial barrier function, on the other hand an anti-inflammatory via the induction of intestinal GC, inhibition of immune cell functions, and repair of epithelial barrier functions. Likely, the orchestrated action of TNF $\alpha$ in time and space may be critical for the dissociation between these pro- and anti-inflammatory properties. 
Tumor-produced GC as a novel mechanism of immune escape?

As discussed above, LRH-1 is not only an essential regulator of intestinal GC synthesis, but has also important functions in promoting epithelial cell renewal and if uncontrolled in the development of intestinal tumors [71]. Thus, LRH-1 may be considered as proto-oncogene. This tumor-promoting effect of LRH-1 is at least in part mediated by the induction of cyclin D1 and E1 expression and uncontrolled cell cycle progression [70]. LRH-1-driven GC synthesis by intestinal tumor cells may, however, further contribute to immune escape of transformed cells and the development of colon carcinoma. It is of interest that the degree of infiltration of colon carcinoma with $\mathrm{T}$ cells negatively correlates with the overall survival of tumor patients [84], suggesting that immune evasion, e.g., via GCmediated suppression of immune cells, may be an effective strategy of intestinal tumors.

We recently tested this hypothesis and assessed the steroidogenic potential of colon carcinoma cells lines and also primary tumors. Interestingly, most colon cancer cell lines and basically all tumor samples expressed LRH-1 and key steroidogenic enzymes required for $\mathrm{GC}$ synthesis (i.e., CYP11A1, CY17, CYP11B1). Furthermore, over-expression of LRH-1 in colon cancer cell lines strongly induced the expression of these steroidogenic enzymes, suggesting that colon carcinomas are capable of synthesizing GC. Indeed, it was found that, in colon cancer cell lines as well as in primary tumors, the synthesis of cortisol can be measured by radioimmunoassay, bioassay, as well as by monitoring the conversion of radiolabeled ${ }^{14} \mathrm{C}$-progesteron to ${ }^{14} \mathrm{C}$-cortisol using thin-layer chromatography (D. Sidler, T. Brunner, unpublished data). Interestingly, when comparing the GC synthesis potential, it was noted that ex vivo culture of colonic tumor samples generally resulted in higher GC concentrations produced than in normal mucosa specimens from the same patient. These findings demonstrate that transformed intestinal epithelial cells, such as colon carcinoma, have maintained the capability to produce GC and further suggest that tumor-derived GC may contribute to cancer development by promoting immune escape.

\section{Conclusions}

The data discussed above clearly demonstrate that the intestinal mucosa and more specifically the intestinal epithelial layer are a complete steroidogenic tissue capable of synthesizing immunoregulatory GC in response to qualitatively distinct immune responses. There is further accumulating evidence that intestinal GC are intimately involved in the regulation of intestinal immune homeostasis and that disruption of critical elements in the GC synthesis pathway in intestinal epithelial cells may lead to an exacerbation of inflammatory responses and increased tissue damage. While the enzymatic cascade and the end product of adrenal and intestinal GC synthesis remain the same, and adrenal and intestinal GC likely also have the same effect on immune cell activation, there is also good evidence that the regulation of intestinal GC synthesis is substantially different from that in the adrenal glands. Two key decision-making molecules appear to be the nuclear receptor LRH-1 and the cytokine TNF $\alpha$. While LRH-1 has been shown to be critical for the transcriptional regulation of steroidogenic enzymes, the direct mechanism of TNF $\alpha$ mediated activation of intestinal steroidogenesis remains to be investigated. The characterization of intestinal GC synthesis leads to the identification of a novel immunoregulatory mechanism in the intestinal mucosa and offers novel targets for therapeutic intervention in the pathogenesis of inflammatory disorders of the intestine.

Acknowledgments This work was supported by the Swiss National Science Foundation, the Crohn's and Colitis Foundation of America, and Oncosuisse. D.S. is recipient of a fellowship from the Swiss National Science Foundation

\section{References}

1. Takahashi I, Kiyono H (1999) Gut as the largest immunologic tissue. JPEN J Parenter Enteral Nutr 23:S7-S12. doi:10.1177/ 014860719902300107

2. Boirivant M, Amendola A, Butera A (2008) Intestinal microflora and immunoregulation. Mucosal Immunol 1(Suppl 1):S47-S49. doi:10.1038/mi.2008.52

3. McGuckin MA, Eri R, Simms LA, Florin TH, Radford-Smith G (2009) Intestinal barrier dysfunction in inflammatory bowel diseases. Inflamm Bowel Dis 15:100-113. doi:10.1002/ibd.20539

4. Lee J, Gonzales-Navajas JM, Raz E (2008) The "polarizingtolerizing" mechanism of intestinal epithelium: its relevance to colonic homeostasis. Semin Immunopathol 30:3-9. doi:10.1007/ s00281-007-0099-7

5. Coombes JL, Powrie F (2008) Dendritic cells in intestinal immune regulation. Nat Rev Immunol 8:435-446. doi:10.1038/nri2335

6. Magalhaes JG, Tattoli I, Girardin SE (2007) The intestinal epithelial barrier: how to distinguish between the microbial flora and pathogens. Semin Immunol 19:106-115. doi:10.1016/j. smim.2006.12.006

7. Macpherson AJ (2006) IgA adaptation to the presence of commensal bacteria in the intestine. Curr Top Microbiol Immunol 308:117-136. doi:10.1007/3-540-30657-9_5

8. Artis D (2008) Epithelial-cell recognition of commensal bacteria and maintenance of immune homeostasis in the gut. Nat Rev Immunol 8:411-420. doi:10.1038/nri2316

9. Kelly D, Conway S, Aminov R (2005) Commensal gut bacteria: mechanisms of immune modulation. Trends Immunol 26:326333. doi:10.1016/j.it.2005.04.008

10. Round JL, Mazmanian SK (2009) The gut microbiota shapes intestinal immune responses during health and disease. Nat Rev Immunol 9(5):313-23 
11. Salzman NH, Underwood MA, Bevins CL (2007) Paneth cells, defensins, and the commensal microbiota: a hypothesis on intimate interplay at the intestinal mucosa. Semin Immunol 19:70-83. doi:10.1016/j.smim.2007.04.002

12. Izcue A, Coombes JL, Powrie F (2006) Regulatory $T$ cells suppress systemic and mucosal immune activation to control intestinal inflammation. Immunol Rev 212:256-271. doi:10.1111/ j.0105-2896.2006.00423.x

13. Becker C, Fantini MC, Neurath MF (2006) TGF-beta as a T cell regulator in colitis and colon cancer. Cytokine Growth Factor Rev 17:97-106. doi:10.1016/j.cytogfr.2005.09.004

14. Powrie F, Correa-Oliveira R, Mauze S, Coffman RL (1994) Regulatory interactions between CD45RBhigh and CD45RBlow $\mathrm{CD} 4+\mathrm{T}$ cells are important for the balance between protective and pathogenic cell-mediated immunity. J Exp Med 179:589-600. doi:10.1084/jem.179.2.589

15. Powrie F, Leach MW (1995) Genetic and spontaneous models of inflammatory bowel disease in rodents: evidence for abnormalities in mucosal immune regulation. Ther Immunol 2:115-123

16. Cima I, Corazza N, Dick B, Fuhrer A, Herren S, Jakob S, Ayuni E, Mueller C, Brunner T (2004) Intestinal epithelial cells synthesize glucocorticoids and regulate $\mathrm{T}$ cell activation. J Exp Med 200:1635-1646. doi:10.1084/jem.20031958

17. Cancedda C, Filaci G, Puppo F, Ghio M, Contini P, Indiveri F (2002) Immune homeostasis requires several biologic factors including glucocorticoid hormones. Ann N Y Acad Sci 966:49-63

18. Wajchenberg BL, Prestes Cesar F, Okada H, Torres de Toledo e Souza I, Lerario AC, Borghi VC, Malerbi DA, Giurna Filho A, Liberman B, Gianella D (1984) Glucocorticoids, glucose metabolism and hypothalamic-pituitary-adrenal axis. Adv Exp Med Biol 171:25-44

19. Berglund M, Thomas JA, Hornquist EH, Hultgren OH (2008) Toll-like receptor cross-hyporesponsiveness is functional in interleukin-1-receptor-associated kinase-1 (IRAK-1)-deficient macrophages: differential role played by IRAK-1 in regulation of tumour necrosis factor and interleukin-10 production. Scand J Immunol 67:473-479. doi:10.1111/j.1365-3083.2008.02096.x

20. Beato M, Herrlich P, Schutz G (1995) Steroid hormone receptors: many actors in search of a plot. Cell 83:851-857. doi:10.1016/ 0092-8674(95)90201-5

21. Kunicka JE, Talle MA, Denhardt GH, Brown M, Prince LA, Goldstein G (1993) Immunosuppression by glucocorticoids: inhibition of production of multiple lymphokines by in vivo administration of dexamethasone. Cell Immunol 149:39-49. doi:10.1006/cimm.1993.1134

22. Almawi WY, Beyhum HN, Rahme AA, Rieder MJ (1996) Regulation of cytokine and cytokine receptor expression by glucocorticoids. J Leukoc Biol 60:563-572

23. Unlap T, Jope RS (1995) Inhibition of NFkB DNA binding activity by glucocorticoids in rat brain. Neurosci Lett 198:41-44. doi:10.1016/0304-3940(95)11963-W

24. Schaaf MJ, Cidlowski JA (2002) Molecular mechanisms of glucocorticoid action and resistance. J Steroid Biochem Mol Biol 83:37-48. doi:10.1016/S0960-0760(02)00263-7

25. De Bosscher K, Vanden Berghe W, Haegeman G (2000) Mechanisms of anti-inflammatory action and of immunosuppression by glucocorticoids: negative interference of activated glucocorticoid receptor with transcription factors. J Neuroimmunol 109:16-22. doi:10.1016/S0165-5728(00)00297-6

26. Scheinman RI, Gualberto A, Jewell CM, Cidlowski JA, Baldwin AS Jr (1995) Characterization of mechanisms involved in transrepression of NF-kappa B by activated glucocorticoid receptors. Mol Cell Biol 15:943-953

27. Karin M (1998) New twists in gene regulation by glucocorticoid receptor: is DNA binding dispensable? Cell 93:487-490. doi:10.1016/S0092-8674(00)81177-0
28. Amsterdam A, Tajima K, Sasson R (2002) Cell-specific regulation of apoptosis by glucocorticoids: implication to their antiinflammatory action. Biochem Pharmacol 64:843-850. doi:10.1016/S0006-2952(02)01147-4

29. Amsterdam A, Sasson R (2002) The anti-inflammatory action of glucocorticoids is mediated by cell type specific regulation of apoptosis. Mol Cell Endocrinol 189:1-9. doi:10.1016/S0303-7207 (01)00722-5

30. Iglesias-Serret D, de Frias M, Santidrian AF, Coll-Mulet L, Cosialls AM, Barragan M, Domingo A, Gil J, Pons G (2007) Regulation of the proapoptotic BH3-only protein BIM by glucocorticoids, survival signals and proteasome in chronic lymphocytic leukemia cells. Leukemia 21:281-287. doi:10.1038/ sj.leu. 2404483

31. Erlacher M, Michalak EM, Kelly PN, Labi V, Niederegger H, Coultas L, Adams JM, Strasser A, Villunger A (2005) BH3-only proteins Puma and Bim are rate-limiting for gamma-radiation- and glucocorticoid-induced apoptosis of lymphoid cells in vivo. Blood 106:4131-4138. doi:10.1182/blood-2005-04-1595

32. Wang Z, Malone MH, He H, McColl KS, Distelhorst CW (2003) Microarray analysis uncovers the induction of the proapoptotic BH3-only protein Bim in multiple models of glucocorticoidinduced apoptosis. J Biol Chem 278:23861-23867. doi:10.1074/ jbc.M301843200

33. Cupps TR, Fauci AS (1982) Corticosteroid-mediated immunoregulation in man. Immunol Rev 65:133-155. doi:10.1111/j.1600065X.1982.tb00431.x

34. Perretti M, D'Acquisto F (2009) Annexin A1 and glucocorticoids as effectors of the resolution of inflammation. Nat Rev Immunol 9:62-70. doi:10.1038/nri2470

35. Kaufman DB, Shapiro R, Lucey MR, Cherikh WS, Bustami RT, Dyke DB (2004) Immunosuppression: practice and trends. Am J Transplant 4(Suppl 9):38-53. doi:10.1111/j.1600-6135.2004.00397.x

36. Di Munno O, Delle Sedie A (2008) Effects of glucocorticoid treatment on focal and systemic bone loss in rheumatoid arthritis. J Endocrinol Invest 31:43-47

37. Simpson ER (1979) Cholesterol side-chain cleavage, cytochrome P450, and the control of steroidogenesis. Mol Cell Endocrinol 13:213-227. doi:10.1016/0303-7207(79)90082-0

38. Bornstein SR, Ehrhart-Bornstein M (2000) Basic and clinical aspects of intraadrenal regulation of steroidogenesis. Z Rheumatol 59 Suppl 2: II:12-17

39. Wang W, Zhang C, Marimuthu A, Krupka HI, Tabrizizad M, Shelloe R, Mehra U, Eng K, Nguyen H, Settachatgul C, Powell B, Milburn MV, West BL (2005) The crystal structures of human steroidogenic factor-1 and liver receptor homologue-1. Proc Natl Acad Sci U S A 102:7505-7510. doi:10.1073/pnas.0409482102

40. Arlt W, Stewart PM (2005) Adrenal corticosteroid biosynthesis, metabolism, and action. Endocrinol Metab Clin North Am 34:293-313. doi:10.1016/j.ecl.2005.01.002 viii

41. Buckingham JC (2006) Glucocorticoids: exemplars of multitasking. Br J Pharmacol 147(Suppl 1):S258-S268. doi:10.1038/ sj.bjp. 0706456

42. Meeking S (2007) Treatment of acute adrenal insufficiency. Clin Tech Small Anim Pract 22:36-39. doi:10.1053/j.ctsap. 2007.02.006

43. Gonzalo JA, Gonzalez-Garcia A, Martinez C, Kroemer G (1993) Glucocorticoid-mediated control of the activation and clonal deletion of peripheral T cells in vivo. J Exp Med 177:12391246. doi:10.1084/jem.177.5.1239

44. Payne AH, Hales DB (2004) Overview of steroidogenic enzymes in the pathway from cholesterol to active steroid hormones. Endocr Rev 25:947-970. doi:10.1210/er.2003-0030

45. Annane D (2005) Glucocorticoids in the treatment of severe sepsis and septic shock. Curr Opin Crit Care 11:449-453. doi:10.1097/ 01.ccx.0000176691.95562.43 
46. Vacchio MS, Ashwell JD (1997) Thymus-derived glucocorticoids regulate antigen-specific positive selection. J Exp Med 185:20332038. doi: $10.1084 /$ jem.185.11.2033

47. Vacchio MS, Lee JY, Ashwell JD (1999) Thymus-derived glucocorticoids set the thresholds for thymocyte selection by inhibiting TCR-mediated thymocyte activation. J Immunol 163:1327-1333

48. Lechner O, Wiegers GJ, Oliveira-Dos-Santos AJ, Dietrich H, Recheis H, Waterman M, Boyd R, Wick G (2000) Glucocorticoid production in the murine thymus. Eur $\mathrm{J}$ Immunol 30:337-346. doi:10.1002/1521-4141(200002)30:2<337::AID-IMMU337>3.0. $\mathrm{CO} ; 2-\mathrm{L}$

49. Pazirandeh A, Xue Y, Rafter I, Sjovall J, Jondal M, Okret S (1999) Paracrine glucocorticoid activity produced by mouse thymic epithelial cells. FASEB J 13:893-901

50. Davies E, MacKenzie SM (2003) Extra-adrenal production of corticosteroids. Clin Exp Pharmacol Physiol 30:437-445. doi:10.1046/j.1440-1681.2003.03867.x

51. Keeney DS, Jenkins CM, Waterman MR (1995) Developmentally regulated expression of adrenal 17 alpha-hydroxylase cytochrome P450 in the mouse embryo. Endocrinology 136:4872-4879. doi:10.1210/en.136.11.4872

52. Brunner T, Arnold D, Wasem C, Herren S, Frutschi C (2001) Regulation of cell death and survival in intestinal intraepithelial lymphocytes. Cell Death Differ 8:706-714. doi:10.1038/sj. cdd. 4400854

53. Atanasov AG, Leiser D, Roesselet C, Noti M, Corazza N, Schoonjans K, Brunner T (2008) Cell cycle-dependent regulation of extra-adrenal glucocorticoid synthesis in murine intestinal epithelial cells. FASEB J 22:4117-4125. doi:10.1096/fj.08114157

54. Mueller M, Cima I, Noti M, Fuhrer A, Jakob S, Dubuquoy L, Schoonjans K, Brunner T (2006) The nuclear receptor LRH-1 critically regulates extra-adrenal glucocorticoid synthesis in the intestine. J Exp Med 203:2057-2062. doi:10.1084/jem.20060357

55. Mueller M, Atanasov A, Cima I, Corazza N, Schoonjans K, Brunner T (2007) Differential regulation of glucocorticoid synthesis in murine intestinal epithelial versus adrenocortical cell lines. Endocrinology 148:1445-1453. doi:10.1210/en.2006-0591

56. Val P, Lefrancois-Martinez AM, Veyssiere G, Martinez A (2003) SF-1 a key player in the development and differentiation of steroidogenic tissues. Nucl Recept 1:8. doi:10.1186/1478-1336-1-8

57. Bland ML, Jamieson CA, Akana SF, Bornstein SR, Eisenhofer G, Dallman MF, Ingraham HA (2000) Haploinsufficiency of steroidogenic factor- 1 in mice disrupts adrenal development leading to an impaired stress response. Proc Natl Acad Sci U S A 97:14488-14493. doi:10.1073/pnas.97.26.14488

58. Fayard E, Auwerx J, Schoonjans K (2004) LRH-1: an orphan nuclear receptor involved in development, metabolism and steroidogenesis. Trends Cell Biol 14:250-260. doi:10.1016/j. tcb.2004.03.008

59. Saxena D, Escamilla-Hernandez R, Little-Ihrig L, Zeleznik AJ (2007) Liver receptor homolog-1 and steroidogenic factor-1 have similar actions on rat granulosa cell steroidogenesis. Endocrinology 148:726-734. doi:10.1210/en.2006-0108

60. Labelle-Dumais C, Jacob-Wagner M, Pare JF, Belanger L, Dufort $\mathrm{D}$ (2006) Nuclear receptor NR5A2 is required for proper primitive streak morphogenesis. Dev Dyn 235:3359-3369. doi:10.1002/ dvdy.20996

61. Coste A, Dubuquoy L, Barnouin R, Annicotte JS, Magnier B, Notti M, Corazza N, Antal MC, Metzger D, Desreumaux P, Brunner T, Auwerx J, Schoonjans K (2007) LRH-1-mediated glucocorticoid synthesis in enterocytes protects against inflammatory bowel disease. Proc Natl Acad Sci U S A 104:13098-13103. doi:10.1073/pnas.0702440104

62. Boivin MA, Ye D, Kennedy JC, Al-Sadi R, Shepela C, Ma TY (2007) Mechanism of glucocorticoid regulation of the intestinal tight junction barrier. Am J Physiol Gastrointest Liver Physiol 292:G590-G598. doi:10.1152/ajpgi.00252.2006

63. Harke N, Leers J, Kietz S, Drenckhahn D, Forster C (2008) Glucocorticoids regulate the human occludin gene through a single imperfect palindromic glucocorticoid response element. Mol Cell Endocrinol 295:39-47. doi:10.1016/j.mce.2008.08.011

64. Kimura T (1986) Transduction of ACTH signal from plasma membrane to mitochondria in adrenocortical steroidogenesis. Effects of peptide, phospholipid, and calcium. J Steroid Biochem 25:711-716. doi:10.1016/0022-4731(86)90299-2

65. Watanabe N, Inoue H, Fujii-Kuriyama Y (1994) Regulatory mechanisms of cAMP-dependent and cell-specific expression of human steroidogenic cytochrome P450scc (CYP11A1) gene. Eur J Biochem 222:825-834. doi:10.1111/j.1432-1033.1994.tb18929.x

66. Rosenberg D, Groussin L, Bertagna X, Bertherat J (2002) cAMP pathway alterations from the cell surface to the nucleus in adrenocortical tumors. Endocr Res 28:765-775. doi:10.1081/ERC-120017071

67. Burendahl S, Treuter E, Nilsson L (2008) Molecular dynamics simulations of human LRH-1: the impact of ligand binding in a constitutively active nuclear receptor. Biochemistry 47:52055215. doi:10.1021/bi7025084

68. Kim YS, Ryu JH, Han SJ, Choi KH, Nam KB, Jang IH, Lemaitre B, Brey PT, Lee WJ (2000) Gram-negative bacteria-binding protein, a pattern recognition receptor for lipopolysaccharide and beta-1, 3-glucan that mediates the signaling for the induction of innate immune genes in Drosophila melanogaster cells. J Biol Chem 275:32721-32727. doi:10.1074/jbc.M003934200

69. Lee YK, Choi YH, Chua S, Park YJ, Moore DD (2006) Phosphorylation of the hinge domain of the nuclear hormone receptor LRH-1 stimulates transactivation. J Biol Chem 281:7850-7855. doi:10.1074/jbc.M509115200

70. Botrugno OA, Fayard E, Annicotte JS, Haby C, Brennan T, Wendling O, Tanaka T, Kodama T, Thomas W, Auwerx J, Schoonjans K (2004) Synergy between LRH-1 and beta-catenin induces G1 cyclin-mediated cell proliferation. Mol Cell 15:499509. doi:10.1016/j.molcel.2004.07.009

71. Schoonjans K, Dubuquoy L, Mebis J, Fayard E, Wendling O, Haby C, Geboes K, Auwerx J (2005) Liver receptor homolog 1 contributes to intestinal tumor formation through effects on cell cycle and inflammation. Proc Natl Acad Sci U S A 102:20582062. doi:10.1073/pnas.0409756102

72. Rutgeerts P (1998) Medical therapy of inflammatory bowel disease. Digestion 59:453-469. doi:10.1159/000007523

73. Cerny A, Chisari FV (1999) Pathogenesis of chronic hepatitis C: immunological features of hepatic injury and viral persistence. Hepatology 30:595-601. doi:10.1002/hep. 510300312

74. Van Assche G, Vermeire S, Rutgeerts P (2008) Optimizing treatment of inflammatory bowel diseases with biologic agents. Curr Gastroenterol Rep 10:591-596. doi:10.1007/s11894-008-0107-7

75. Neurath MF, Fuss I, Pasparakis M, Alexopoulou L, Haralambous S, Meyer zum Buschenfelde KH, Strober W, Kollias G (1997) Predominant pathogenic role of tumor necrosis factor in experimental colitis in mice. Eur J Immunol 27:1743-1750. doi:10.1002/eji.1830270722

76. Naito Y, Takagi T, Handa O, Ishikawa T, Nakagawa S, Yamaguchi T, Yoshida N, Minami M, Kita M, Imanishi J, Yoshikawa T (2003) Enhanced intestinal inflammation induced by dextran sulfate sodium in tumor necrosis factor-alpha deficient mice. J Gastroenterol Hepatol 18:560-569. doi:10.1046/j.1440-1746.2003.03034.x

77. Ebach DR, Newberry R, Stenson WF (2005) Differential role of tumor necrosis factor receptors in TNBS colitis. Inflamm Bowel Dis 11:533-540. doi:10.1097/01.MIB.0000163698.34592.30

78. Rutgeerts P, Vermeire S, Van Assche G (2009) Biological therapies for inflammatory bowel diseases. Gastroenterology 136:1182-1197. doi:10.1053/j.gastro.2009.02.001

79. Kassiotis G, Kollias G (2001) Uncoupling the proinflammatory from the immunosuppressive properties of tumor necrosis factor 
(TNF) at the p55 TNF receptor level: implications for pathogenesis and therapy of autoimmune demyelination. J Exp Med 193:427-434. doi:10.1084/jem.193.4.427

80. Zheng L, Fisher G, Miller RE, Peschon J, Lynch DH, Lenardo MJ (1995) Induction of apoptosis in mature $T$ cells by tumour necrosis factor. Nature 377:348-351. doi:10.1038/377348a0

81. Zhou T, Edwards CK III, Yang P, Wang Z, Bluethmann H, Mountz JD (1996) Greatly accelerated lymphadenopathy and autoimmune disease in lpr mice lacking tumor necrosis factor receptor I. J Immunol 156:2661-2665
82. Matthys P, Billiau A (1997) Cytokines and cachexia. Nutrition 13:763-770. doi:10.1016/S0899-9007(97)00185-8

83. Clayburgh DR, Barrett TA, Tang Y, Meddings JB, Van Eldik LJ, Watterson DM, Clarke LL, Mrsny RJ, Turner JR (2005) Epithelial myosin light chain kinase-dependent barrier dysfunction mediates $\mathrm{T}$ cell activation-induced diarrhea in vivo. J Clin Invest 115:2702-2715. doi:10.1172/JCI24970

84. Rubin EM, Raptopoulos VD (2005) Images in clinical medicine. The virtual apple core of a colonic carcinoma. N Engl J Med 352:2733. doi:10.1056/NEJMicm040235 